OUARTERLY PROGRESS REPORT

\title{
SUPERCRITICAL THERMODYNAMICS OF SULFUR AND NITROGEN SPECIES
}

Project No. DE-FG22-91PC91287

Principal Investigator:

Initiation Date:

Period Covered by Report:
Prof. Charles A. Eckert

Georgia Institute of Technology

School of Chemical Engineering

(404) 853 - 9344

October 1, 1991

October 1, 1992 - December 31,1992

\section{ABSTRACT}

Significant opportunity exists for the application of supercritical fluid (SCF) technology to coal processing, both for pretreatment of high sulf 2 r coals, as well as liquefaction and treatment of coal liquids. Supercritical fluids are attractive solvents for a variety of coal processing applications because of their unusual solvating and mass transfer properties. Solubility studies have been carried out for a number of model coal and coal-liquid compounds, primarily in pure supercritical fluids. We are extending this database of model coal compound equilibria using modern techniques that have the advantage of being much more rapid than traditional techniques. Cosolvent effects on solubility are being investigated over a variety of solvent properties. In addition, specific molecular interactions are being investigated through spectroscopic techniques. The resulting data are being used to develop a chemical-physical equation of state (EOS) model of SCF solution with meaningful parameters. The equation of state will be used to predict solubility behavior, which will permit the design and tailoring of SCF cosolvent systems for specific coal processing applications.

\section{DISCLAIMER}

This report was prepared as an account of work sponsored by an agency of the United States This employees, makes any warranty, express or implied, or assumes any legal liability or responsibility for the accuracy, completeness, or usefulness of any information, apparatus, product, or process disclosed, or represents that its use would not infringe privately owned rights. Reference herein to any specific commercial product, process, or service by trade name, trademark, manufacturer, or otherwise does not necessarily constitute or imply its endorsement, recommendation, or favoring by the United States Government or any agency thereof. The views and opinions of authors expressed herein do not necessarily state or reflect those of the United States Government or any agency thereof.

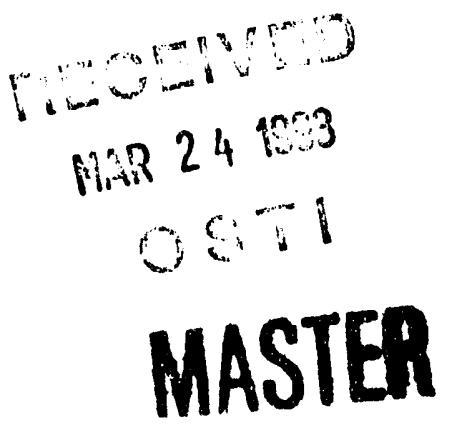

der 


\section{OBJECTIVES}

The focus of this work is the application of supercritical fluid (SCF) solvents to the precombustion desulfurization and denitrogenization of coal, as well as to the liquefaction and subsequent processing of coal liquids. In particular, we focus on the design of solvent-cosolvent systems for treating nitrogen and sulfur heterocycles. A novel technique using supercritical fluid chromatography measures rapidly the cosolvent effect on solubility and is used to extend the existing database of solubility

data. Further details of molecular interactions in supercritical fluids, including specific solvation and the "clustering phenomena" in the near critical region, are investigated using high-pressure spectroscopic experiments. Data obtained from these experiments will be used in the development of a chemical-physical equation of state that accounts for the unique solvation properties of supercritical fluid solutions using accurate and chemically-meaningful parameters.

Supercritical fluids (SCF) show potential for coal processing because they possess some unique properties. A SCF is quite dense, which gives a large capacity for solutes; it has high diffusivity and low viscosity, which makes it an ideal medium for efficient mass transfer; and the fluid is highly compressible, which yields large solubility changes with small changes in temperature and pressure. The addition of a small amount of cosolvent (usually less than $5 \%$ ) can dramatically enhance solubility. These phenomena suggest extraordinary selectivity possibilities. Therefore, we are proceeding with the measurement of solubilities in these novel fluids and fluid mixtures.

Solubility data exist for a wide variety of chemical compounds for pure solid solutes in several pure SCF's. The majority of these data are taken in SCF systems with a low critical point. Solubility data has recently been extended to include some heavier compounds typical of coal structures and solubilities in supercritical fluids with higher critical temperatures. These data have been correlated with a variety of cubic equations of state (EOS's) and semitheoretical expressions. However, in order to account for the effect of cosolvent on solubility and exploit potential selectivities in model coal compound systems, an EOS developed though a molecular approach is necessary.

Our ongoing studies of cosolvent effects both support the development of a chemical-physical EOS as well as extend the existing database of solubilities of model coal compounds in SCF solutions. The supercritical fluid chromatography technique permits rapid measurement of these effects. The solvent, cosolvent and solute are carefully chosen to gain maximum information on the relative importance of solute and cosolvent properties such as polarity, acidity, and basicity on phase equilibria. 
Direct spectroscopic measurements of the specific interactions which yield extreme solubility and cosolvent effects will also be used to understand the relative importance of specific interactions in SCF's. The spectroscopic phenomena investigated include charge-transfer complexing, hydrogen bonding, and dipole coupling. A high-pressure UV-vis spectrometer and a high-pressure fiuorescence spectrometer are being used to probe these interactions. From spectral features, the strength of solute/solute and solute/solvent interactions in the near-critical region can be quantified. Both the solubility and spectroscopy data are vital to the development of an equation of state model that will take into account both the physical and chemical forces that are important in SCF solutions. 


\section{PROGRESS THIS QUARTER}

\section{PHASE EQUILIBRIA AND COSOLVENT EEEECTS}

Under past DOE support, we compiled an extensive database of coal model compound solubilities. Although cosolvent-modified SCF's show much promise in facilitating difficult separations, relatively little data exist on the effects of these modifiers. Both the magnitude and the importance of specific molecular interactions on cosolvents effects are yet unknown. Therefore, we are now extending this solubility database by using two novel techniques for measuring solubilities in supercritical fluid solutions containing cosolvents. Through carefully designed experiments, we are also working to delineate the effects of various specific interactions on model coal compounds solubilities in SCF solutions.

One reason that solubility and cosolvent data are scarce is that conventional techniques to measure solubilities are time consuming and require large amounts of solute. A chromatographic technique that we developed to measure these thermodynamic phenomenon in SCF solutions is attractive because chromatographic measurements are rapid, require little solute, and inherently separate impurities from the solute. With this technique, we are measuring solubilities and cosolvent effects from the retention time of the solute in the column using the SCF solution of interest as the mobile phase. We define the cosolvent effect, $\Psi$, for a solute as the ratio of its solubility in the fluid mixture to the solubility in the pure fluid at the same temperature and pressure. We have demonstrated the viability of this technique (Ekart et al., 1992); the absolute solubilities measured using this technique compare favorably with literature data.

In a recently completed study of the role of specific interactions on cosolvent effects, Kamlet-Taft solvatochromic parameters (Kamlet and Taft, 1976; Kamlet et al., 1977, 1983), dipole moments, and polarizabilities guided our cosolvent selection. Cosolvents possessing either relatively extreme hydrogen bond donating abilities or hydrogen bond accepting abilities were of particular interest to our investigation. Solubilities and cosolvent effects were measured for several solutes, again chosen for their ability to participate in various specific interactions. Although hydrogen bonding apparently contributes to cosolvent effects, our results also show that other solute/cosolvent interactions such as charge transfer complexing, dipole/dipole alignment and polarity/polarizibilities can be exploited to improve selectivities and loadings. In order to understand the contributions of these effects better, a wider range of cosolvents must be studied. 
Our current cosolvent studies involve a series of small cosolvent molecules with only minor perturbations in structure. These cosolvents vary widely, not only in their ability to hydrogen bond, but in polarity, polarizability, and Lewis acidity/basicity. The solutes, shown in Figure 1, are a homologous series of fluorene and anthracene compounds. Nitrogen-containing compounds and sulfur compounds such as these are also of interest to coal processing. All studies are conducted in SCF mixtures of ethane, which does not participate in specific interactions with the cosolvents or solutes, modified with 3.5 mole\% cosolvent. Minor perturbations in chemical structure of both the cosolvent and solute will allow us to study a variety of specific interactions

High pressure VLE data with ethane is currently not available for these cosolvents. However, knowledge of phase boundaries is necessary at the mixing bomb temperature $\left(25^{\circ} \mathrm{C}\right.$ ) and column temperature $\left(50^{\circ} \mathrm{C}\right)$ to ensure operation within the one phase region. Therefore, we have determined a near-critical phase boundary for each $3.5 \%$ cosolvent/ethane mixture. The conditions of these phase boundaries are given in Table 1 . Table 1 also notes the miscibility of each cosolvent with room temperature liquid ethane at its vapor pressure. This information guides our choice of mixing bomb and column operating conditions.

Following this preliminary cosolvent screening, we are measuring the chromatographic retention times for every solute in given SCF mixture. The comparative technique used to determine cosolvent effects from chromatographic data requires retention times of a single solute in several SCF mixtures. We are currently collecting the data needed for this comparison for the solute/cosolvent/SCF ethane systems previously described.

\section{SPECIFIC INTERACTIONS THROUGH SPECTROSCOPY}

Our recent investigation of cosolvent effects using a chromatographic technique (above) has shown that specific interactions other than hydrogen bonding also contribute to the enhanced solubilities of solutes in SCF cosolvent systems. Spectroscopic techniques are suited to quantifying the extent of many of these contributing interactions, particularly charge transfer complexing. From a series of experiments in our double-beam UV-vis spectrometer adapted for high-pressure applications, the equilibrium constant of the charge transfer complex can be calculated. These equilibrium constants are integral to the development of predictive chemical-physical equations of state. In addition, knowledge of equilibrium constants in the near-critical region as a function of density provides insight into the local environment of these solute molecules.

As discussed in a previous report, we are determining the equilibrium constants of the charge transfer complex of hexamethylbenzene (HMB) and tetracyanoethylene (TCNE) in SCF ethane. Ethane was chosen as our SCF fluid for 
the same reasons that it is used in our cosolvent studies: it has moderate critical properties and does not participate in specific interactions with our electron donor or acceptor species. TCNE is a powerful electron-withdrawing species which accepts a pi electron from HMB. The complex formed by this interaction, which has been well-studied in liquid solutions, absorbs light in a region about $250 \mathrm{~nm}$ from the reactants. In addition, the molar extinction coefficient is sufficiently large that the complex can be observed in even dilute solutions. An absorption spectrum for this complex and components in liquid $\mathrm{CH}_{2} \mathrm{Cl}_{2}$ is given in Figure 2.

A Benesi-Hildebrand approach is used to determine equilibrium constants from the measured UV intensities of the complex (Eqn. 1). This approach is only applicable when the mole fraction of HMB is much greater than that of TCNE. This analysis also assumes that the absorption of the complex follows Beer's law. Unlike the standard Benesi-Hildebrand equation, the equilibrium constant is found in pressure-independent mole-fraction units for a nonideal system. A thermodynamic model is required to calculate absolute equilibrium constants. The equilibrium constant $\left(\mathrm{K}_{\mathrm{x}}\right)$ and extinction coefficient $(\varepsilon)$ at a specific solvent density are determined from the slope and intercept of a linear plot.

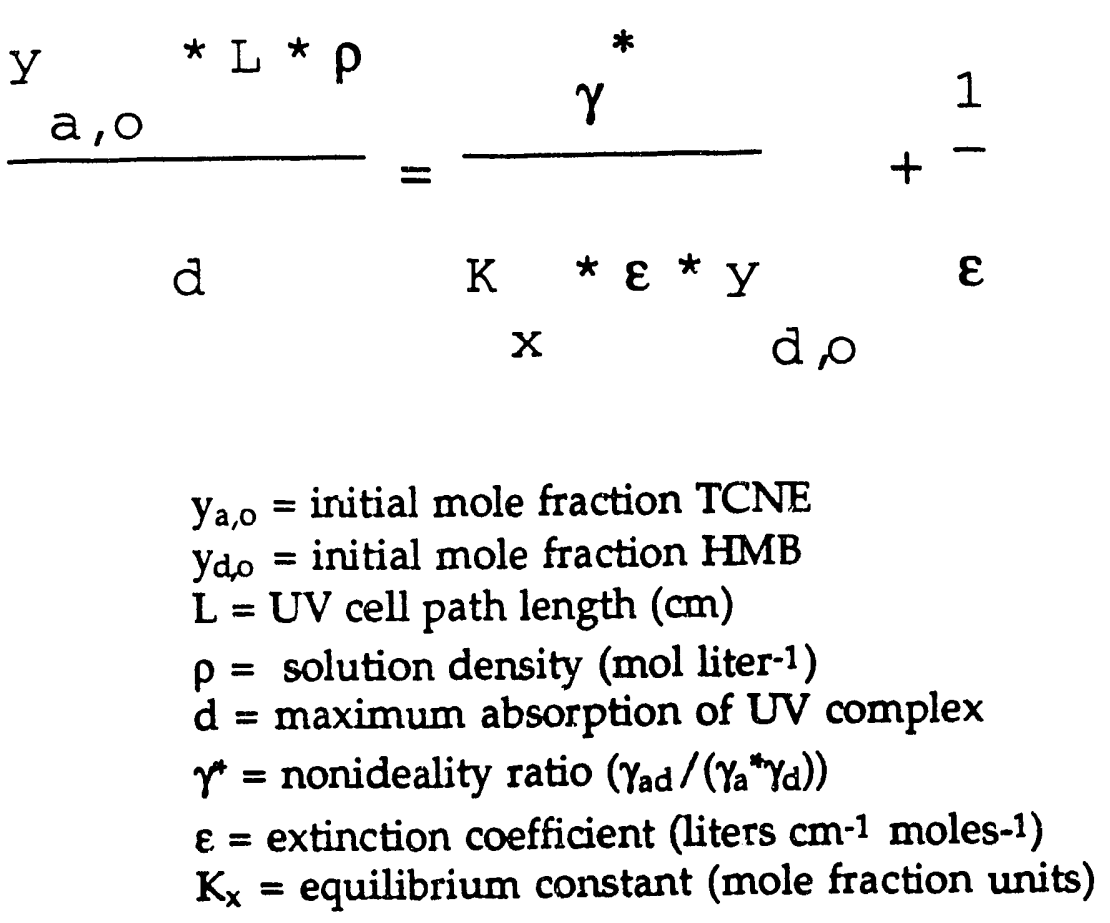

(Eqn. 1)

A sample Benesi-Hildebrand analysis for the TCNE/HMB/SCF ethane system at a density of 11.5 moles/liter is shown in Figure 3. The initial concentrations of TCNE and HMB were varied at constant solution density and path 
length. The maximum absorption of the UV complex was measured, and the nonideality ratio was estimated. The equilibrium constant and complex extinction coefficient are than determined from a linear plot. Figure 4 gives the resulting equilibrium constants at several solution densities. These equilibrium constants are consistent with those determined in liquids (Ewald, 1968) both in magnitude and in pressure effects.

Complete analysis of these results requires a comprehensive comparison of our measured equilibrium constants with those measured in liquids at high pressures, as well as fine-tuning of our thermodynamic model. In addition, we plan to measure the solubility of TCNE in pure SCF ethane and SCF ethane modified with HMB. We will then study the feasibility of modelling this cosolvent effect using our measured equilibrium constants in a chemical-physical equation of state.

\section{PLANS FOR NEXT QUARTER}

We have measured cosolvent effects on solubility for the SCF/cosolvent/solute systems using a chromatographic technique. The solvents, cosolvents, and solutes were carefully chosen to gain maximum understanding of the importance of specific chemical interactions, in particular hydrogen bonding, on cosolvent effects. The results of this study suggest that other specific interactions also contribute to hydrogen bonding. We have completed the initial screening of a new series of cosolvents with only mild perturbations in structure. These cosolvents vary widely, not only in their ability to hydrogen bond, but in polarity, polarizability, and Lewis acidity/basicity. Currently, we are measuring cosolvent effects in these SCF ethane/cosolvent/solute systems.

We have measured charge transfer complex formation in SCF solutions over a range of densities using a Benesi-Hildebrand approach. Next quarter we plan to fine-tune the thermodynamic model associated with this analysis as well as compare these results with equilibrium constants in liquid systems as a function of pressure. We will measure the cosolvent effect, or the solubility increase due to charge transfer complexing, in the TCNE/HMB cosolvent/SCF ethane system.

We will continue testing and improving our new chemical-physical equation of state based upon our solubility, cosolvent, and spectroscopic data. Equilibrium constants, such as those measured spectroscopically for charge transfer complexes, are potential chemically-meaningful parameters in this equation of state. 


\section{SUMMARY}

Supercritical fluids show potential for a variety of coal processing applications. Application of SCF technology to these processes is limited by a dearth of information on the phase equilibria of coal compounds in SCF solutions and cosolvent effects on these solubilities. We are taking a molecular-based approach to developing and improving a chemical-physical equation of state with chemicallymeaningful parameters. The goal of this work is the ability to tailor SCF cosolvent systems to specific coal processing applications through knowledge of the chemical interactions which are significant to the SCF system.

Future work will include further investigations of cosolvent effects using supercritical fluid chromatography, fluorescence spectroscopy, and UV absorption. This information and the database of solubility measurements will be used in the further development of an equation of state model to predict solubilities for the design of coal treatment processes.

\section{REFERENCES}

Ekart, M.P., Bennett, K.L.,; Eckert, C.A., "A Chromatographic Technique to Study the Effects of Specific Interactions in Supercritical Fluid Solutions," submitted to ACS Sym. Ser., 1992.

Ewald, A.H., "Effect of Pressure on Charge Transfer Complexes in Solution," Trans. Farad. Soc., 1968, 64, 733.

Johnston, K.P.; Kim, S., "Clustering in Supercritical Fluid Mixtures," AIChE J., 1987, 33,1603 .

Kamlet, M.J.; Taft, R.W., "The Solvatochromic Comparison Method. 1. the $\beta$-Scale of Solvent Hydrogen-Bond Acceptor (HBA) Basicities," J. Am. Chem. Soc., $1976,98,377$.

Kamlet, M.J.; Taft, R.W., "The Solvatochromic Comparison Method. 6. The $\pi^{*}$ Scale of Solvent Polarities," J. Am. Chem. Soc., 1977, 99, 6027.

Kamlet, M.J.; Abboud, J.L.; Abraham, M.H.;Taft, RW., "Linear Solvation Energy Relationships. 23. A Comprehensive Collection of the Solvatochromic Parameters, $\pi^{*}, \alpha, \beta$ and Some Methods for Simplifying the Generalized Solvatochromic Equation," J. Org. Chem., 1983, 48, 2877. 
Table 1. Near-critical phase boundaries of $3.5 \mathrm{~mole} \%$ cosolvent/ethane solutions. The miscibility of 3.5 mole\% cosolvent with liquid ethane at its room temperature vapor pressure is also noted with a $\mathrm{Y}$ (miscible) or $N$ (immiscible).

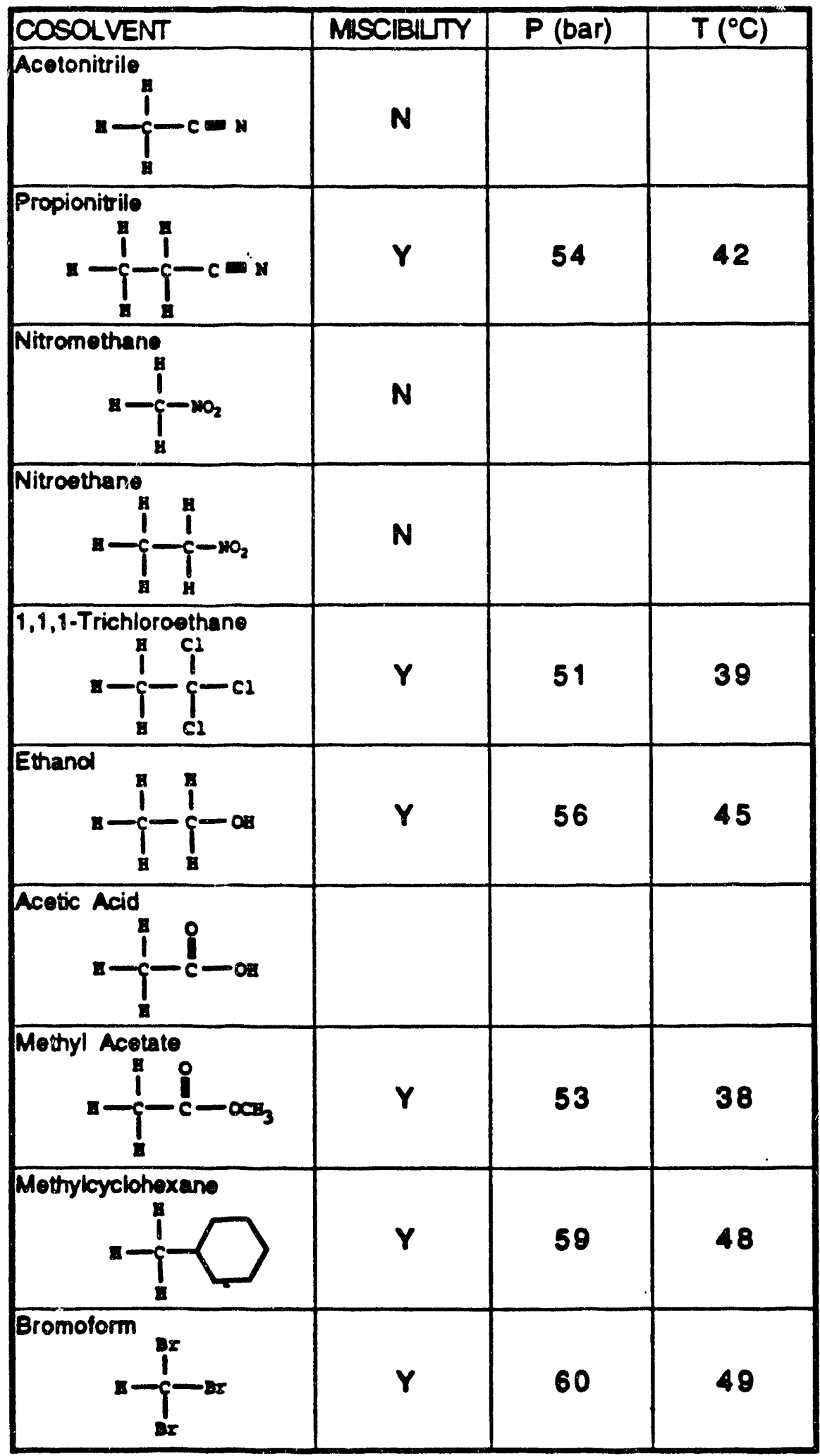




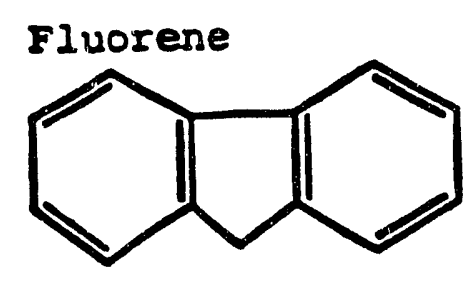

Dibenzofuran<smiles>c1ccc2c(c1)oc1ccccc12</smiles>

Dibenzothiophene<smiles>c1ccc2c(c1)sc1ccccc12</smiles>

Carbazole<smiles>[Z3]n1c2ccccc2c2ccccc21</smiles>

Fluorenone<smiles>O=C1c2ccccc2-c2ccccc21</smiles>

9-Hydroxy Eluorene<smiles>OC1c2ccccc2-c2ccccc21</smiles>

Anthracene<smiles>c1ccc2cc3ccccc3cc2c1</smiles>

9-Cyanoanthracene

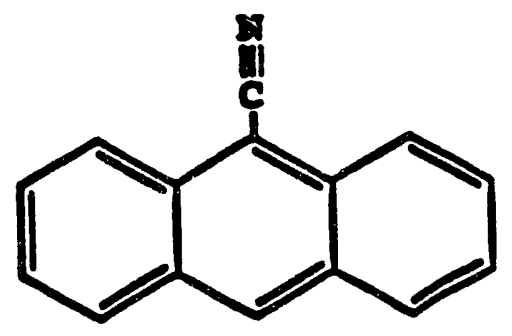

Phenazine<smiles>c1ccc2nc3ccccc3nc2c1</smiles>

Thianthrene<smiles>c1ccc2c(c1)Sc1ccccc1S2</smiles>

9,10-Dihydroxyanthracene<smiles>CC1c2ccccc2C(O)c2ccccc21</smiles>
Anthracuinone<smiles>O=C1c2ccccc2C(=O)c2ccccc21</smiles>

Figure 1. Series of solutes. 


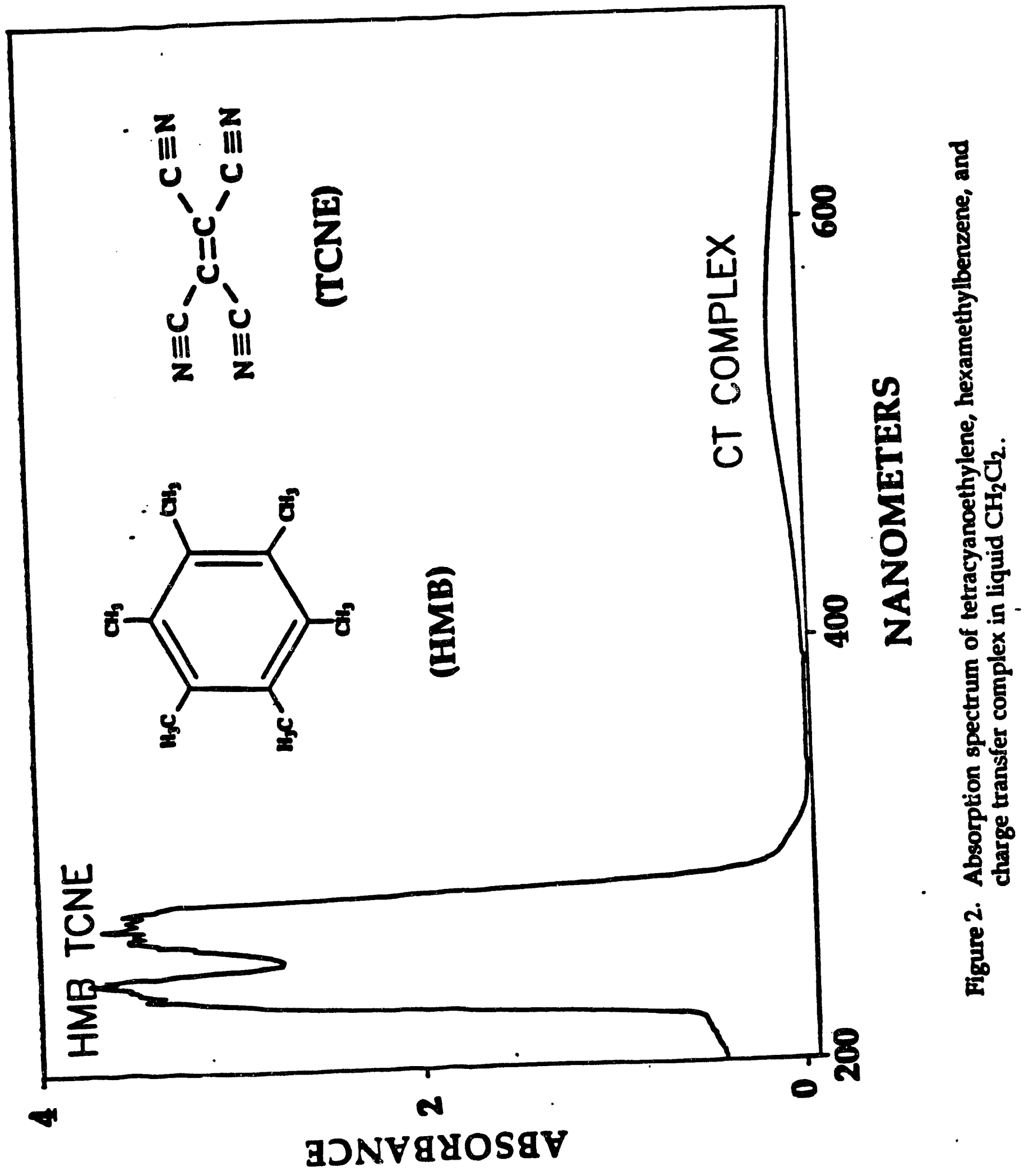



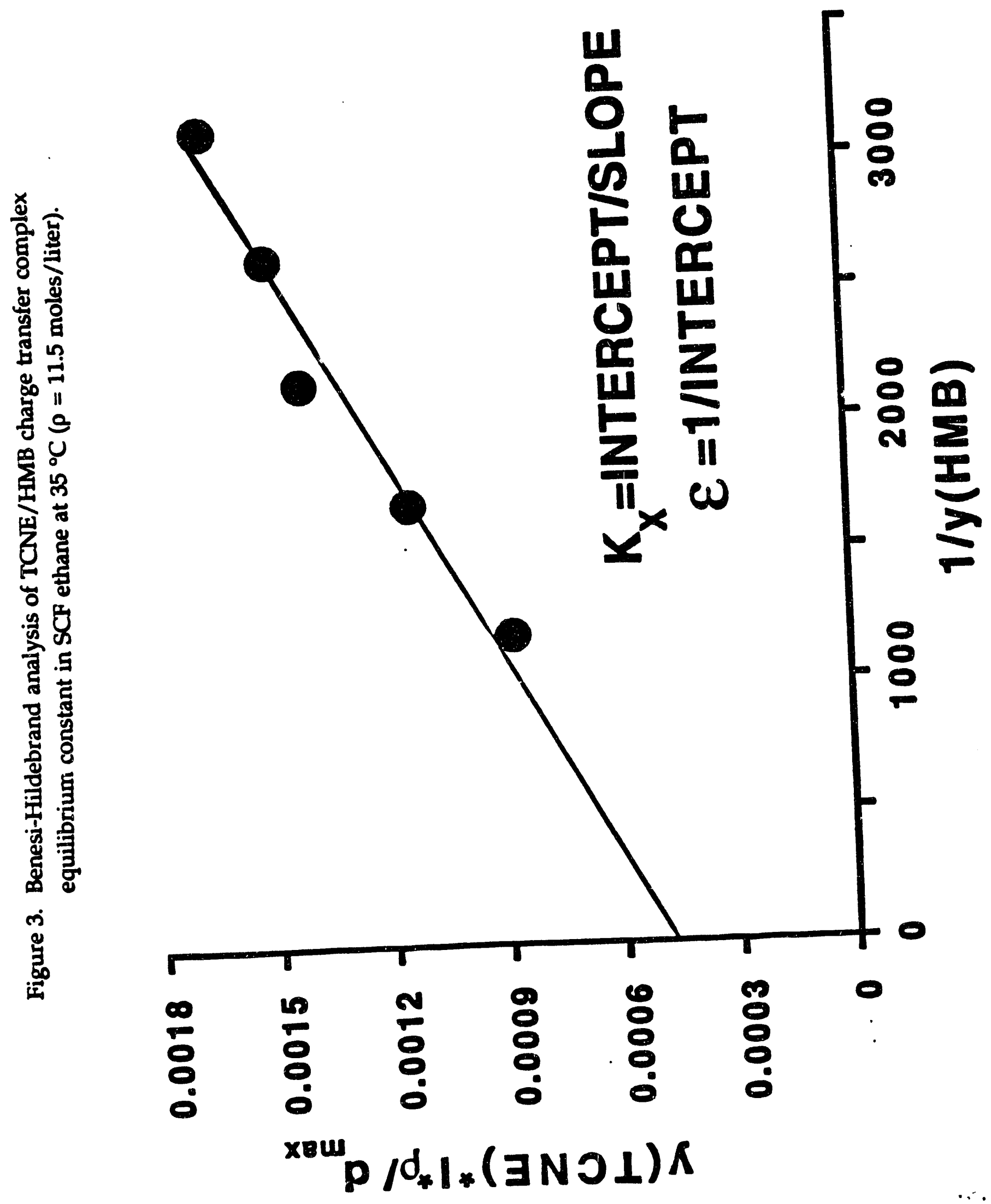


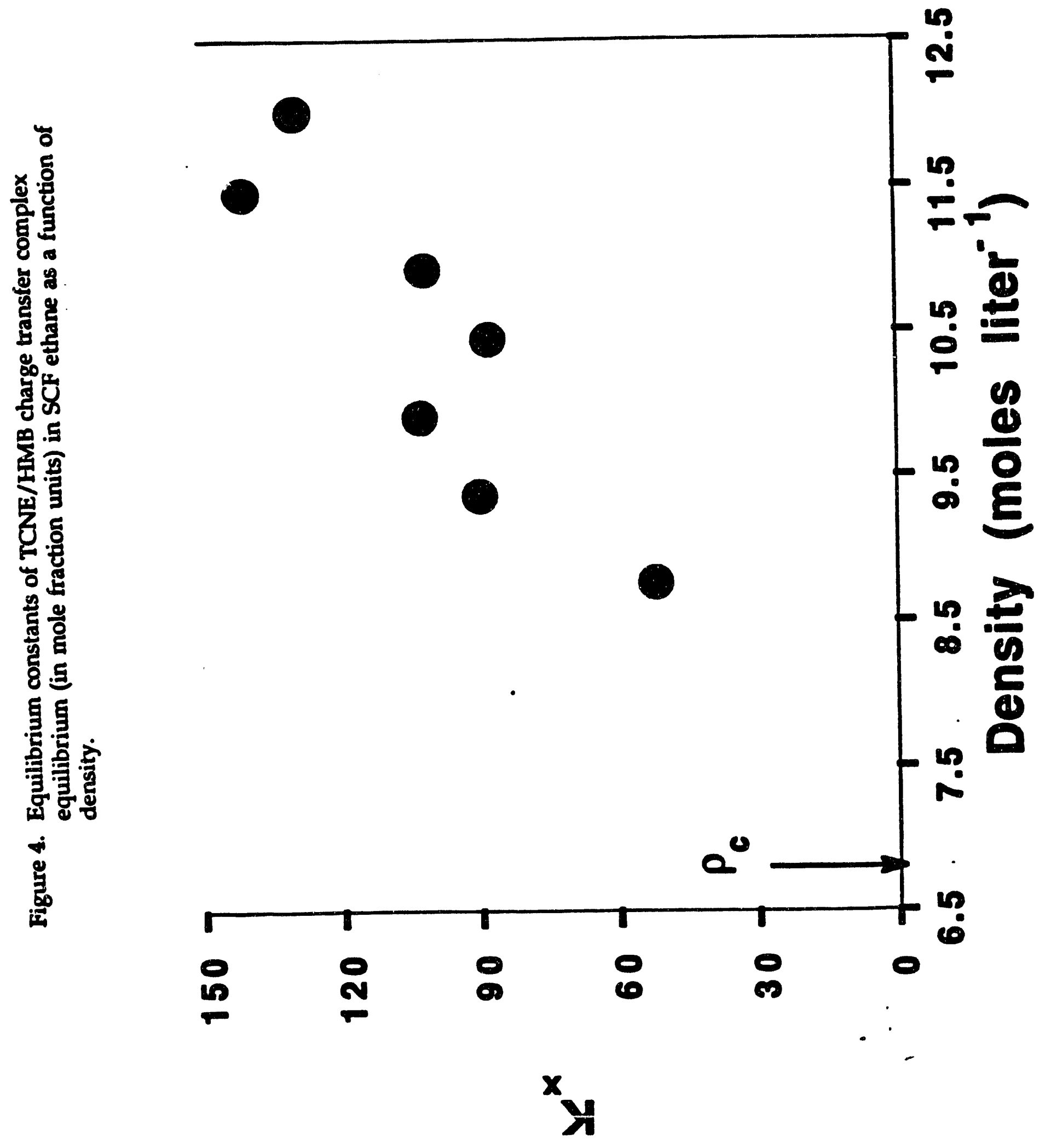



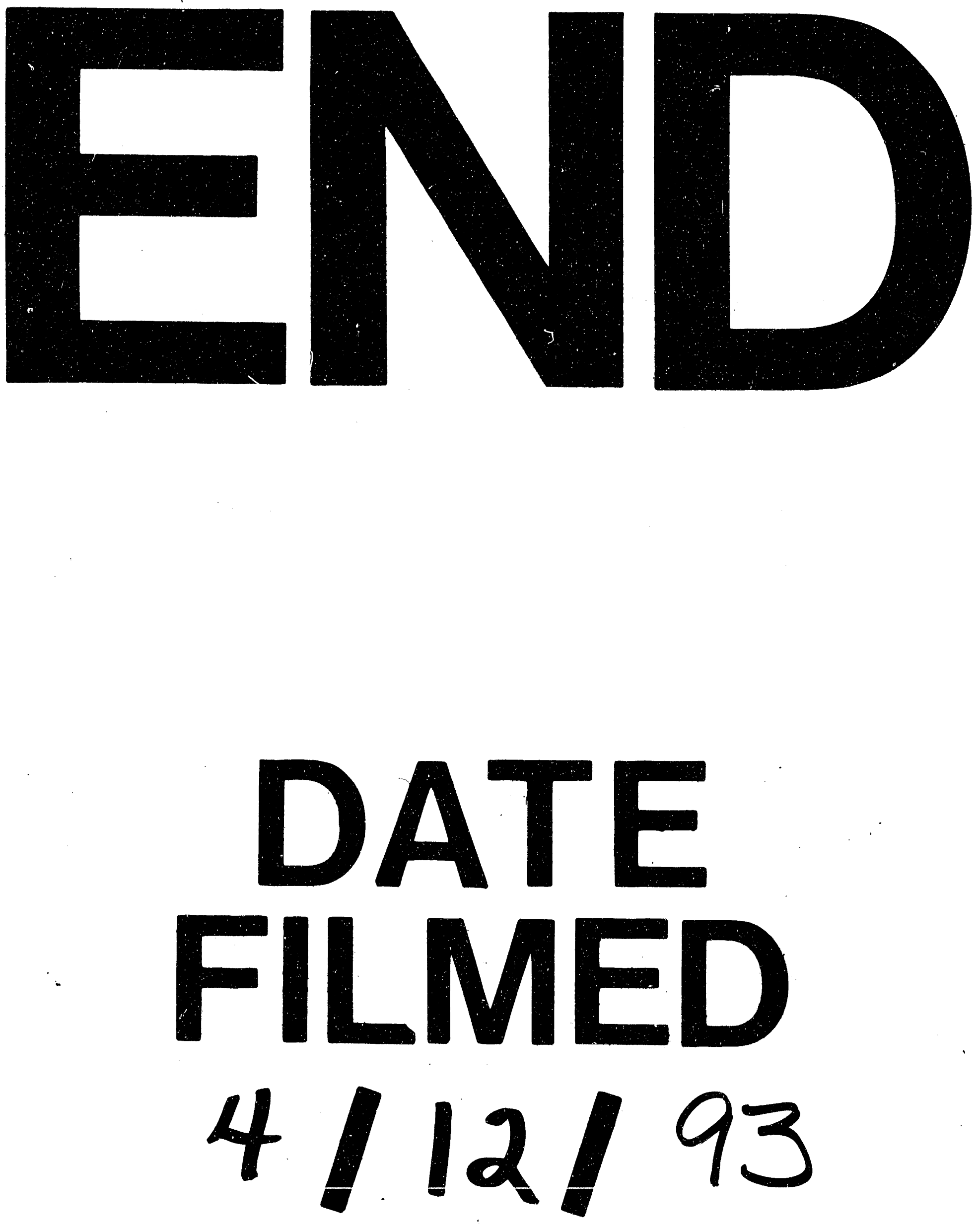
